Revue scientifique francophone en Communication

organisationnelle

$18 \mid 2000$

Non-verbal, communication, organisation

\title{
Du rituel chez les immortels
}

\author{
Claude Brévot Dromzée
}

URL : https://journals.openedition.org/communicationorganisation/2474

DOI : 10.4000/communicationorganisation.2474

ISSN : $1775-3546$

\section{Éditeur}

Presses universitaires de Bordeaux

\section{Édition imprimée}

Date de publication : 1 novembre 2000

ISSN : 1168-5549

\section{Référence électronique}

Claude Brévot Dromzée, "Du rituel chez les immortels », Communication et organisation [En ligne], 18 | 2000, mis en ligne le 19 décembre 2012, consulté le 05 août 2021. URL : http://

journals.openedition.org/communicationorganisation/2474; DOI : https://doi.org/10.4000/

communicationorganisation.2474

Ce document a été généré automatiquement le 5 août 2021.

(c) Presses universitaires de Bordeaux 


\title{
Du rituel chez les immortels
}

\author{
Claude Brévot Dromzée
}

Depuis 1635, une institution se consacre "corps et âme» à la défense et à la diffusion de la langue: l'Académie française. Indépendamment du cliché se situe «le corps académique ». entité dont la fonction est définie dans les textes fondateurs de l'organisation. "L'âme» de ce corps institutionnel est la loi de l'usage, référence explicitement citée dans chacune des Préfaces du Dictionnaire et motif récurrent lors des séances académiques.

2 Sous la Coupole de l'Institut de France, le verbe est roi. L'élaboration séculaire du Dictionnaire (la neuvième édition est en cours) témoigne de l'intérêt - durable - que les Académiciens peuvent porter à la langue. La tradition du discours académique relève de la « littérature d'apparat » selon Marc Fumaroli. Si celle-ci, à l'époque classique, se définit comme "l'art de bien dire», quelle peut être la place du non-verbal dans l'institution académique? En quoi ce non-verbal co-construit-il la représentation de l'Institution à travers un événement annuel : la séance publique annuelle? En quoi ce cérémonial - représentant l'identité visuelle de l'Institution - est-il aussi vecteur de communication?

3 Revenons d'abord à la genèse de l'Académie.

\section{Construction d'un système}

4 Ni société de gens de lettres, ni société savante, l'Académie française définit sa fonction ainsi : il s'agit de «donner des regles certaines à nostre langue et la rendre pure, éloquente, capable de traiter les arts et les sciences $»^{1}$. Cette profession de foi s'explique en raison d'un contexte encore très latinisant (notamment dans les inscriptions officielles) et cela près d'un siècle après l'Ordonnance de Villers-Cotterêts (1539). La langue étant " parvenuë à ce glorieux point d'immutabilité » dont il est question dans la Dédicace de la première édition du Dictionnaire (1694), il incombe aux amateurs de Belles-Lettres réunis autour de Conrart vers 1630 , de constituer une compagnie officielle veillant sur la défense et l'illustration de la langue. C'est l'initiative de Richelieu qui crée ainsi un organe de propagande - susceptible de propager la foi - 
《instrument du faire-croire $»^{2}$. Il s'agit de faire comme si la langue constituait un objet d'étude autonome et monolithique, conférant à l'Académie autorité de droit et indépendance de fait.

Or si l'amour de la langue française a dicté un tel projet, la tentation d'une hégémonie linguistique - régir la langue au même titre que d'autres secteurs - et la volonté d'affirmer une autorité politique ont sans doute motivé la création de l'Académie. La même année (1635) Richelieu fonde l'Institution et déclare la guerre à l'Espagne. En ce qui concerne l'Académie, il s'agit d'un acte de politique linguistique, voire de planification linguistique, que l'Institution s'approprie en créant le concept de l'usage.

6 La place grandissante, dans les discours de réception, de l'éloge du Roi - aux dépens de celui du récipiendaire - fait apparaître la véritable fonction de l'Académie : organe de communication du pouvoir. C'est d'ailleurs ce symbole que fustige la Révolution française - et pas directement sa fonction lexicographique - en démantelant l'Académie en 1793. tel en témoigne le Discours Préliminaire ${ }^{3}:$ « instituée, protégée par des Ministres, par des Rois, dont les éloges revenoient incessamment dans tous ses discours, on eût dit que l'unique et servile objet de la fondation [de l'Académie] étoit l'art de cacher la bassesse de la flatterie sous les vains agrémens de la parole ».

7 Puisque le Dictionnaire peut se définir comme «un outil linguistique politiquement finalisé [...] le rôle de la définition [étant] en concurrence avec celui d'une représentation culturelle de la langue $"^{4}$. c'est la justification même de sa création - la langue - qui fait de l'Académie un lieu de représentation, un lieu de fabrication de représentation : choisir, nommer, célébrer.

\section{S'inscrire dans la durée}

L'éloge royal s'inscrit dans le genre éminemment pratiqué à l'Académie: le panégyrique - «suture symbolique entre les vivants et les morts [...] il est au centre de toute fête qui rend une société présente à elle-même, et la rassemble autour d'une patrie et d'un patrimoine commun et sacré $»^{5}$. Au-delà de l'expression d'une certaine quintessence rhétorique, il joue un rôle social comme facteur de cohésion tu sein du corps académique; il co-construit le paradigme de la durée, assurant depuis plus de trois siècles ce continuum de la «belle langue ». Autre constante du paradigme: le principe d'immutabilité de la langue affirmé dès la première édition du Dictionnaire; c'est la confrontation de ce principe lié à la règle de l'usage qui justifie la succession des éditions du Dictionnaire au fil... très distendu du temps ! Suprême facette du paradigme : la notion d'immortalité. Elle est, depuis l'origine, inscrite dans les statuts (article 2: "L'Académie aura un sceau [...] et un contre-sceau où sera représentée une couronne de laurier, avec ce mot : A l'immortalité ») ; elle est métaphoriquement l'appellation des gardiens de la langue qui sont amenés à transcender leur condition d'humains : les académiciens deviennent « immortels»!

9 Certes la notion de durée est ici poussée à son paroxysme, suscitant parallèlement quolibets et sarcasmes - les académiciens se savent couverts de brocarts et... de brocards; « ceux qui reprennent [ces flèches] et les relancent n'aperçoivent peut-être pas qu'ils se placent par là dans la tradition comme nous. Nos moqueurs nous sont consubstantiels " écrit l'auteur du Cimetière marin ${ }^{6}$. Mais toute institution ne s'inscritelle pas d'abord dans la durée, faite d'éléments invariants, comme le constate Marc 
Abélès dans sa récente étude de l'Assemblée nationale: «vieille dame bicentenaire, celle-ci semble traverser les siècles sans être affectée par le temps qui passe [...] le président incarne la pérennité de la représentation nationale ${ }^{7}$.

\section{Réception ou Intronisation?}

10 Les signes sont ostensibles et récurrents, particulièrement lors des séances publiques qui rythment l'année académique et qui constituent ainsi une sorte de ponctuation de la durée. Si 1635 est la date de fondation de l'Académie française, celle-ci prend véritablement forme avec l'instauration de la séance publique, quelques décennies plus tard. Ainsi la voix des orateurs se théâtralise, c'est le début d'une mise en scène verbale et surtout non-verbale, faite de "marqueurs co-textuels et contextuels $»^{8}$ qui vont ponctuer la vie académique. L'actuelle carte de vœux de l'Académie représente «la réception d'un académicien", gravure de Poilly d'après De la Monce; cette représentation iconographique semble illustrer un compte rendu (séance du 30 octobre 1718) contenu dans le Mercure galant ${ }^{9}$ : « celui qu'on reçoit est assis au bord d'en bas de la table, parce que n'ayant pas encore de place dans l'Académie, il semble qu'il ne la doive prendre qu'après sa Réception. Le Directeur est vis-à-vis de lui à l'autre bout de la Table, assis dans un fauteuil. Les officiers sont à ses costez. et le reste de l'Académie sur des chaises autour de la Table » derrière eux, debout, le public. Le récipiendaire, ici, est à la fois «dedans » - la table assurant le lien - et encore en dehors : il s'apprête à subir le « rite de passage » qui va l'intégrer à la compagnie, sur le mode du partage (cum-panis). La cérémonie de la réception (on parle d'« installation " pour l'Académie des BeauxArts) suit un rituel dont l'ordonnancement est différent de la séance annuelle. Dans le premier cas, c'est une affaire de personnes: à la tête du cortège se rendant à la cérémonie se tient celui qui reçoit (l'homme sans nom mais non sans qualités !) puis le directeur en exercice, le Secrétaire perpétuel et, derrière, le récipiendaire encadré de ses parrains. L'espace est ainsi structuré hiérarchiquement et chronologiquement, celui qui est reçu - en dernier - est aussi accueilli par ceux qui l'encadrent et le précèdent. Pour la séance publique, c'est le discours qui est roi avec la prééminence du genre panégyrique. Ainsi le cortège se compose d'abord de l'auteur du discours sur la vertu, puis du directeur, enfin du maître des lieux - le Secrétaire perpétuel.

11 Ce rituel est une composante de la notion de cérémonie qui s'applique (lit-on dans la première édition de 1694) «aux choses humaines, et se dit de certaines formalités qu'on observe dans les actions solennelles pour les rendre plus éclatantes ». Du fait du caractère public de la séance et de l'enjeu de son contenu (accueil d'un nouveau membre ou accueil d'une nouvelle année académique), ce rituel - personnel ou collectif - doit se lire au-delà de la formalité, du protocole.

Qu'en est-il aujourd'hui de la séance annuelle au sein de l'Académie?

\section{Le jour de la rentrée}

13 Elle a lieu invariablement le dernier jeudi de novembre ou le même jour de la semaine suivante. Rentrée différée de plusieurs mois depuis la fondation de l'Académie. D'abord fixée le 25 août, jour de la Saint Louis, et donnant lieu à un hommage appuyé au protecteur Souverain, la séance publique s'est symboliquement libérée de la contingence calendaire (saint François? saint Jacques ?) célébrant son protecteur - le 
chef de l'État pour ne conserver qu'un lien métonymique avec l'appareil gouvernemental : la garde républicaine.

Rentrée officielle pour la doyenne des cinq académies qui composent l'Institut de France, la séance est « publique » dans la mesure des places disponibles (environ quatre cents sous la Coupole) et sur invitation! Trois discours se succèdent: sur les prix littéraires, sur l'état de la langue, sur la vertu. La séance est donc à la fois couronnement de fin d'année et « rentrée de la classe de français » avec le Discours sur l'état de la langue prononcé par le Secrétaire perpétuel. Sur environ trois cent prix décernés par l'Institution et cités par le duc de Castries $^{10}$ en 1978, beaucoup ont été regroupés en prix de vertus - attribués à des personnes "dont le dévouement va de pair avec le dénuement $»^{11}$ - prix (de genres) littéraires et depuis 1986, le Grand Prix de la Francophonie.

La lecture du Palmarès s'articule autour de la notion de mérite, pilier du corps académique. Dire le mérite des lauréats, c'est justifier le choix de l'Institution, c'est donner la preuve de son propre mérite. Effet de miroir, forme d'auto-célébration propre à toute institution: "Lorsqu'un orateur parle devant une institution (qu'il y appartienne ou non), celle-ci attend de lui qu'il développe les valeurs qu'elle cautionne et qu'il donne d'elle une image qui la satisfasse $»^{12}$.

Le Discours sur l'état de la langue relève de la responsabilité du Secrétaire perpétuel, conformément à l'article 24 des Statuts et Règlements de 1635. Posture du vigile de la langue, dont le rapport garantit symboliquement la pérennité de l'Institution.

D'un point de vue rhétorique, la première allocution s'inscrit dans le genre démonstratif (ou épidictique). le discours sur la vertu dont se charge le président de la séance s'apparente plutôt au genre délibératif qui vise, selon Aristote. à persuader : il s'agit ici d'user de son talent d'amateur de belle langue pour haranguer l'auditoire en faveur de la vertu, ultime message de la cérémonie et rappel des bases morales du corps académique.

\section{L'habit vert}

Si l'identité du corps académique cherche à se placer entre deux pôles d'excellence linguistique et morale - celle de ses membres s'estompe plusieurs fois l'an sous l'habit vert, signe d'appartenance le plus manisfeste - avec l'épée et le bicorne - à l'Institution. Même si le port du costume est rare («marqueur» des séances publiques) et partiellement observé (parfois à peine un quart des quarante académiciens le portent !), c'est ce signe-introduit par Bonaparte au début du siècle dernier - qui « en dit le plus » sur le rituel académique. Le costume est d'abord la marque du rite de passage. Confectionné aux frais du récipiendaire pour la cérémonie de réception, il signifie pour la famille présente - à la fois biologique et académique - un engagement solennel. Il affiche une symbolique de l'immortalité redondante : rameaux d'olivier en broderie sur le plastron, couleur verte du frac, «d'un vert particulier, savant et pédagogique, acide et rigide, un vert d'abat-jour, de drap de bureau et de reliure de dictionnaire » ironise Henri Lavedan. académicien du début du siècle ${ }^{13}$. L'habit vert est aussi le signe expressif d'intégration au groupe dans ses valeurs sociétales d'unité et d'égalité, conformément aux Statuts, Toute nouvelle réception d'un académicien "répare » la disparition de l'ancien membre grâce à la permanence du costume, élément construisant le paradigme de la durée. Dispositif représentatif du sentiment 
d'appartenance au corps académique, le costume est aussi symbolique d'une mise à distance de ce qui est "séculier »: l'Académie peut se définir comme "une espèce pensante qui ne tient pas tant à se reproduire, elle accepte avec contentement de grands délais et de grands vides... nul n'a pouvoir sur elle car elle se sait durable $»^{14}$.

La tradition de l'épée est aussi une initiative napoléonienne, désignant chez les fonctionnaires d'autorité, la force liée à "l'imperium », héritage monarchique qui s'est maintenu aujourd'hui en se transformant. Le pommeau de la canne initialement prévue portait en effigie la médaille de l'Institut; aujourd'hui il s'agit souvent d'un objet artistique personnalisé, évoquant par un lien métonymique la carrière du récipiendaire. Accessoire rituel dont sont dispensés les ecclésiastiques. L'épée auraitelle donc une âme ? La réception à l'Académie d'un membre de l'Eglise ne peut rappeler un rite de passage médiéval - l'adoubement - au cours duquel un ecclésiastique arme un nouveau chevalier en lui remettant une épée !

L'autre accessoire dont est dispensée une partie du corps académique (les femmes) est le bicorne... "d'encaisseur de banques", selon la formule de Jean Dutourd. Souci de coquetterie ou règle implicite? " la singularité de l'Académie est d'être indéfinissable », écrit Valéry ${ }^{15}$.

\section{Les trois $P$}

21 Marc Abélès évoque pour l'Assemblée nationale ${ }^{16}$ « les trois $P$ : protocole, patrimoine, précédent ». Si l'Académie ne peut avoir le culte du précédent, elle se caractérise par l'éclat d'une certaine pompe, tant à travers la récurrence du panégyrique, du costume, des accessoires que de la présence de la garde républicaine lors des séances publiques. Retentissement des tambours et présentation des armes ponctuent l'entrée et la sortie des Académiciens sous la Coupole, soulignant la solennité du moment, réitérant ainsi tout ce qui a fait date dans l'Histoire - des sacres aux exécutions publiques, de la pompe royale aux pompes funèbres!

Si le protocole désigne l'ensemble des règles à observer en matière de préséances dans les cérémonies officielles, la séance publique met en scène des formes protocolaires signifiantes. L'ordonnancement du cortège, la succession des discours, la disposition des orateurs constituent des motifs rituels, dont la fonction est pragmatique pour construire cette situation de communication et qui s'enchaînent selon un schéma narratologique précis.

23 Le troisième P. - comme patrimoine - se décline dans le rapport à l'espace : le Collège des Quatre Nations devenu l'Institut de France par un décret napoléonien en 1795. Illustres jalons associés aujourd'hui à «la vieille dame du quai Conti». icônes des strates de l'Histoire : Mazarin le fondateur du lieu, symboliquement présent par son cénotaphe et par ses armes (lanières et faisceau de licteur) sculptées au tambour de la Coupole ; Le Vau maître d'œuvre du lieu; Louis XIV. le protecteur installant la compagnie au Louvre jusqu'à ce qu'une décision révolutionnaire mette fin à l'existence des académies. La Coupole est d'abord une chapelle, affectation marquée par un verset d'Ezechiel toujours présent sur la corniche; actuellement ce lieu est doublement connoté - laïc et républicain, appelé parfois de façon officielle Parlement du monde savant, souvent dénommé Académie française plutôt qu'Institut. Appellation impropre liée davantage au rang qu'elle occupe implicitement auprès de ses consœurs plutôt qu'à son ancienneté. 
Bien qu'elle soit ovale à l'intérieur, la Coupole offre une disposition semi-circulaire des sièges, auxquels fait face le pupitre des orateurs. La parole circule d'un orateur à l'autre, établissant avec l'assistance (spatialement ordonnée: les lauréats, les académiciens puis les accompagnateurs et invités) un réseau de représentations à travers la langue. Être sous la Coupole, c'est être sous la Langue, promue, défendue et illustrée au cours de la séance publique.

Si sous la Coupole, le verbe est souverain au point de constituer depuis plus de trois cent cinquante ans l'unique objet de tous les soins des académiciens, le rituel est un rouage essentiel de la vie académique et de sa représentation. À l'image de la circularité de la Coupole, la composition de l'Académie (quarante membres) semble immuable, sans variation intérieure, sans distinction ou division - une certaine idée de la perfection. Plus que la proclamation verbale, c'est le non-verbal - par des «signes communicatifs et expressifs » - qui met en scène et représente les principes d'unité, d'égalité à l'intérieur du cercle académique d'une part, de pérennité de l'Institution d'autre part.

Si le rituel sert à représenter l'Académie, quel contenu celle-ci peut-elle communiquer à l'extérieur sans être prise au piège de l'obsolescence, voire de la caricature? Que signifie un gros plan de télévision sur les ors et les brocarts d'un « habit vert » à la sortie d'une séance publique? Quelle serait l'efficacité d'une image s'attardant sur le nécessaire débat que suscite l'élaboration du Dictionnaire, sur l'éloquence d'un orateur à la tribune ? "figure anachronique " $\left.{ }^{17}\right)$ écrivait-on déjà il y a quinze ans. Être ou paraître? la question ne peut être tranchée puisqu'elle se pose aussi au sein de l'Institution, qui oscille entre "le non-événement» (les séances de travail) et l'événement (les séances publiques), entre opacité et ostentation.

À chacun sa représentation. Comment parler de l'Académie? est-ce cet ensemble de " rites [pratiqués dans] la cathédrale du quai Conti ${ }^{18}$ ? est-ce cette " tribu qui campe là depuis troi siècles et demi avec des plumes sur la tête, la tribu des hommes verts ${ }^{19}$ ? Le dernier mot revient à l'auteur de la notion de «divertissement». qui ne fit pas - lui partie de la «tribu» : «La douceur de la gloire est si grande qu'à quelque objet qu'on l'attache, même à la mort, on l'aime $»^{20}$.

\section{NOTES}

1. Daniel Oster, Histoire de l'Académie française, Paris, Vialetay,1970, (Statuts et Règlements de l'Académie françoise : 1635, article 24) $194 \mathrm{p}$.

2. Michèle Fogel, Les cérémonies de l'information dans la France du seizième au milieu du dix-huitième siècle, Fayard, 1989, $438 \mathrm{p}$.

3. Bernard Quemada (sous la direction de), les Préfaces du Dictionnaire de l'Académie française 1694-1992, Paris Champion, 1997, 564 p.

4. Simone Delesalle, Francine Mazière, «Raison, foi et usage. Les modes de la signification dans le Dictionnaire de l'Académie (1694) la Grammaire générale et raisonnée et la Logique de Port-Royal ", Sémiotiques. $\mathrm{n}^{\circ} 14$, pp .15-68. 
5. Marc Fumaroli, «Les mots, la Coupole », in Pierre Nora (dir.) Les lieux de mémoire, II, vol. 3, La Nation, Paris, Gallimard, 1986, 1034 p.

6. Paul Valéry, Regards sur le monde actuel, la Pléiade, 1945, 381 p.

7. Marc Abélès, Un ethnologue à l'Assemblée, éd. Odile Jacob, janv. 2000, 283 p.

8. Jacques Cosnier, Les voies du langage : communications verbales, gestuelles et animales, Dunocl. 1982, $329 \mathrm{p}$.

9. cité dans A. Fabre, Chapelain et nos deux premières académies, 1890, $514 \mathrm{p}$.

10. Castries (duc de), La vieille dame du quai Conti. Une histoire de l'Académie française, Librairie académique Perrin, 1978.

11. Pierre Moinot, Directeur en exercice lors la séance publique annuelle du 30 novembre 1995, Rapport sur les prix littéraires.

12. Pierre Zoberman, «Eloquence d'apparat et représentation institutionnelle: pouvoir du magistrat et autorité de l'orateur ", Romanic Review, vol. LXXIX, mars 88, n 2, p. 262.

13. Henri Lavedan. habit vert. 1911, Firmin Didot, 89 p.

14. Choucri Cardahi, Regards sous la Coupole, Mame, 1966, 445 p.

15. Paul Valéry, op. cit.

16. Marc Abélès, op. cit.

17. Jean Starobinski, «La chaire, la tribune, le barreau », In P. Nora (dir.), Les lieux de mémoire, II, La Nation, Gallimard, 1986. Cité par Marc Abélès (op. cit.), p. 238.

18. Marc Fumaroli, op. cit.

19. Jean Dutourd, cité dans André Damien, L 'Institut de France, 1999.

20. Blaise Pascal, Pensées, Garnier, 1991.

\section{AUTEUR}

\section{CLAUDE BRÉVOT DROMZÉE}

Université de Reims Champagne-Ardenne 